Article

\title{
Transcriptome Analysis Revealed the Embryo-Induced Gene Expression Patterns in the Endometrium from Meishan and Yorkshire Pigs
}

\section{Jiangnan Huang ${ }^{1,2}$, Ruize Liu ${ }^{1}$, Lijie Su ${ }^{1}$, Qian Xiao ${ }^{1}$ and Mei Yu ${ }^{1, *}$}

1 Key Lab of Agricultural Animal Genetics, Breeding, and Reproduction of Ministry and the Cooperative Innovation Center for Sustainable Pig Production, Huazhong Agricultural University, Wuhan 430070, China; E-Mails: huangjiangnan@webmail.hzau.edu.cn (J.H.); 1rz_2003@163.com (R.L.); sulijie@webmail.hzau.edu.cn (L.S.); xiaoqian2013@sjtu.edu.cn (Q.X.)

2 Institute of Animal Husbandry and Veterinary, Jiangxi Academy of Agricultural Sciences, Nanchang 330200, China

* Author to whom correspondence should be addressed; E-Mail: yumei@mail.hzau.edu.cn; Tel.: +86-27-8728-1306.

Academic Editor: Kotb Abdelmohsen

Received: 26 July 2015 / Accepted: 14 September 2015 /Published: 18 September 2015

\begin{abstract}
The expression patterns in Meishan- and Yorkshire-derived endometrium during early (gestational day 15) and mid-gestation (gestational days 26 and 50) were investigated, respectively. Totally, 689 and 1649 annotated genes were identified to be differentially expressed in Meishan and Yorkshire endometrium during the three gestational stages, respectively. Hierarchical clustering analysis identified that, of the annotated differentially expressed genes (DEGs), 73 DEGs were unique to Meishan endometrium, 536 DEGs were unique to Yorkshire endometrium, and 228 DEGs were common in Meishan and Yorkshire endometriums. Subsequently, DEGs in each of the three types of expression patterns were grouped into four distinct categories according to the similarities in their temporal expression patterns. The expression patterns identified from the microarray analysis were validated by quantitative RT-PCR. The functional enrichment analysis revealed that the common DEGs were enriched in pathways of steroid metabolic process and regulation of retinoic acid receptor signaling. These unique DEGs in Meishan endometrium were involved in cell cycle and adherens junction. The DEGs unique to Yorkshire endometrium were associated with regulation of Rho protein signal transduction, maternal placenta development and cell proliferation. This study revealed the different gene expression patterns or pathways related
\end{abstract}


to the endometrium remodeling in Meishan and Yorkshire pigs, respectively. These unique DEGs in either Meishan or Yorkshire endometriums may contribute to the divergence of the endometrium environment in the two pig breeds.

Keywords: gene expression pattern; pig; endometrium; Meishan and Yorkshire pigs; microarray

\section{Introduction}

A major limitation for increasing the litter size in pigs is the prenatal mortality that mainly occurs during the time of attachment (around gestational day (GD) 12-25) and mid-gestation (day 50-70 of gestation) [1,2]. The multiparous Chinese Meishan pigs had a 20\%-34\% greater prenatal survival than the less prolific Yorkshire pigs [3-6]. It is generally accepted that a greater number of embryo survival in prolific Meishan pigs is due, in part, to more gradual changes in the uterine milieu and greater uterine capacity [7-11].

The pig is a species with a non-invasive placenta and the uterine luminal epithelium (LE) is intact throughout pregnancy [12,13]. The embryo implantation process is accompanied with transition in uterine luminal epithelial cell polarity from a high to less polar state and secretion of glandular epithelium (GE) [14-16]. At the time of implantation, the conceptuses produce estrogen, which acts as a signal for maternal recognition of pregnancy in pigs [17]. Porcine conceptuses secrete increased levels of estrogens on Days 11 and 12 and between Days 15 and 25 of pregnancy [18]. The estrogen, type I interferons (IFNG) and type II interferons (IFND) from the conceptuses together with the progesterone (P4) can temporally and spatially regulate the endometrial gene expression to determine the functional and structural changes in uterine cells [19-21]. After GD30, embryo loss are thought to result from the intrauterine crowding in swine caused by the limitations in uterine capacity [22]. Chinese Meishan pigs exhibit physiological strategy by markedly increasing in number of the uterine glands or vascular density and permeability to overcome potential limits in uterine capacity [4,11]. The functional difference between Meishan and Yorkshire uteri is due to the differences in endometrial structural modifications [23,24]. Thus, gaining an understanding of the molecular mechanisms underlying the role of the uterine endometrium remodeling during pregnancy would be an important step towards investigating the molecular basis of the sow prolificacy.

Some studies which were conducted on the investigation of the gene expression profiles in the porcine endometrium during the early gestation phase were published [12,25-34]. However, investigation of the genes with different expression patterns in endometrium of pigs with prolificacy (Meishan pigs) and less prolificacy (Yorkshire pigs) is an important step towards understanding the mechanism of the uterine function in embryo development and survival. The aim of this study was to identify the differentially expressed genes in endometrium between GD15 and GD26 and between GD26 and GD50 in Meishan and Yorkshire pigs, respectively. We found the common differentially expressed genes (DEGs) in Meishan and Yorkshire gilts related to the signaling networks and pathways which could be essential for endometrial remodeling in response to pregnancy during the early and mid-gestation. Moreover, the identified DEGs unique to Meishan and Yorkshire gilts, respectively, will be useful to investigate the different mechanisms involved in supporting the embryo implantation and growth. 


\section{Results}

\subsection{Detection of the Differentially Expressed Genes}

In pigs, prenatal mortality occurs in several stages of gestation and mainly results from the early embryo mortality and the uterine capacity limits [35]. In order to identify genes expressed in endometrium that may affect the porcine embryo implantation and placentation, the gene expression profiles on GD15, GD26 and GD50 in endometriums of Meishan and Yorkshire gilts were examined, respectively. GD15 and GD26 represent the middle and end of the peri-implantation period (approximately Days 12-25 of pregnancy), respectively. On GD50, the attachment between the pig conceptus trophoblasts and the endometrial epithelial cells is finished and the pig placenta is in a steady-state stage of development [36]. We performed two comparisons (GD26 vs. GD15 and GD50 vs. GD26) within each breed, respectively. Taking a FC $\geq 2$ and the adjusted $p$-value $\leq 0.05$ significance level as the criteria, totally, 689 and 1649 annotated genes (DEGs) showed differential expression in endometrium of Meishan and Yorkshire breeds at the three gestational stages, respectively (Tables S1 and S2). Of the 689 DEGs in Meishan endometrium, 641 genes were found to be differentially expressed between GD15 and GD26 and 79 genes were found to be differentially expressed between GD26 and GD50. Of the 1649 DEGs in Yorkshire endometrium, 1598 genes were found to be differentially expressed between GD15 and GD26 and 91 genes were found to be differentially expressed between GD26 and GD50 (Figure 1). All data related to this study has been deposited on the GEO database (Accession number GSE51787). By hierarchical clustering analysis, we found that 228 DEGs were common in Meishan and Yorkshire gilts, which were likely associated with the embryo implantation and placentation (Table S3), a total of 73 DEGs were unique to Meishan gilts (Table S4) and 536 DEGs were unique to Yorkshire gilts (Table S5).

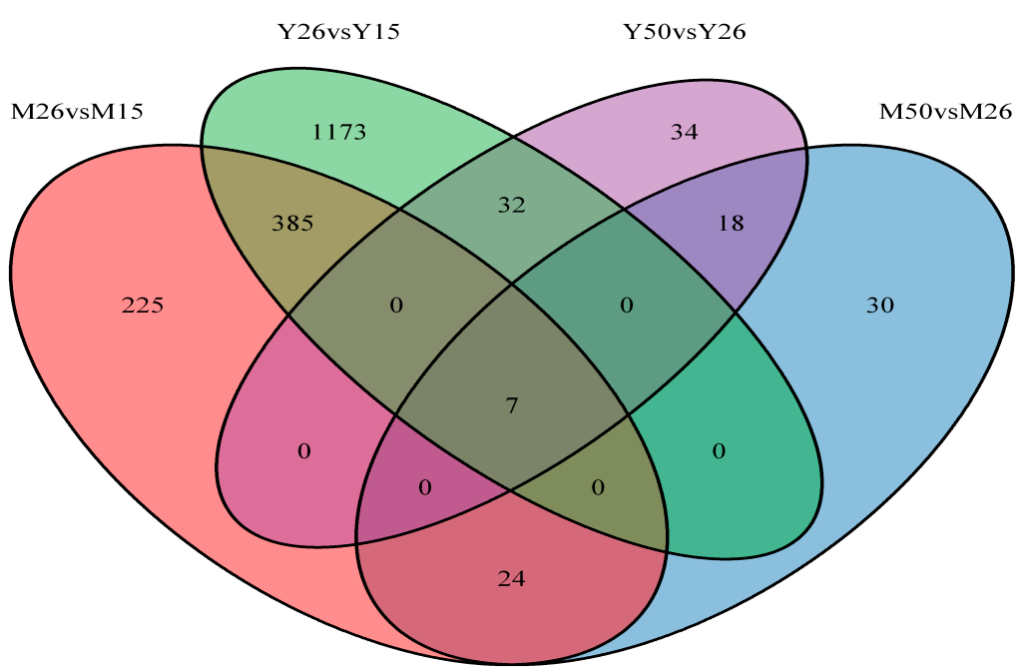

Figure 1. Venn diagram illustration of endometrial genes changed between days 15 and 26 of gestation and days 26 and 50 of gestation in Meishan and Yorkshire pigs. M15, Meishan pigs on day 15 of gestation. M26, Meishan pigs on day 26 of gestation. M50, Meishan pigs on day 50 of gestation. Y15, Yorkshire pigs on day 15 of gestation. Y26, Yorkshire pigs on day 26 of gestation. Y50, Yorkshire pigs on day 50 of gestation. DEGs as defined by FDR $<0.05$ and $\mathrm{FC} \geq 2$. 


\subsection{Identification of Genes Required for the Embryo Implantation and Placentation in}

\section{Porcine Endometrium}

The unsupervised hierarchical clustering analysis revealed that the 228 annotated common DEGs in Meishan and Yorkshire samples were classified into four groups (Figure 2A). (I) Expression level decreased only from GD15 to GD26 and thereafter remained constant ( $n=167$ genes, group 1); (II) Expression level only increased from GD26 to GD50 ( $n=21$ genes, group 2); (III) Expression level increased from GD15 to GD26 and thereafter remained constant ( $n=23$ genes, group 3$)$; and (IV) Increased from GD15 to GD26, then decreased sharply on GD50 ( $n=17$ genes, group 4). The 167 genes in group 1 were enriched for GO terms related to steroid metabolic process, oxidation reduction, response to insulin stimulus, and regulation of retinoic acid receptor signaling pathway. Genes that are involved in negative regulation of cell proliferation and regulation of phosphorylation were found in group 2. The genes in group 3 were enriched for GO terms involved in steroid biosynthesis and genes that regulate the inflammatory response and cell proliferation were in group 4 (Figure 2B, Table S6).

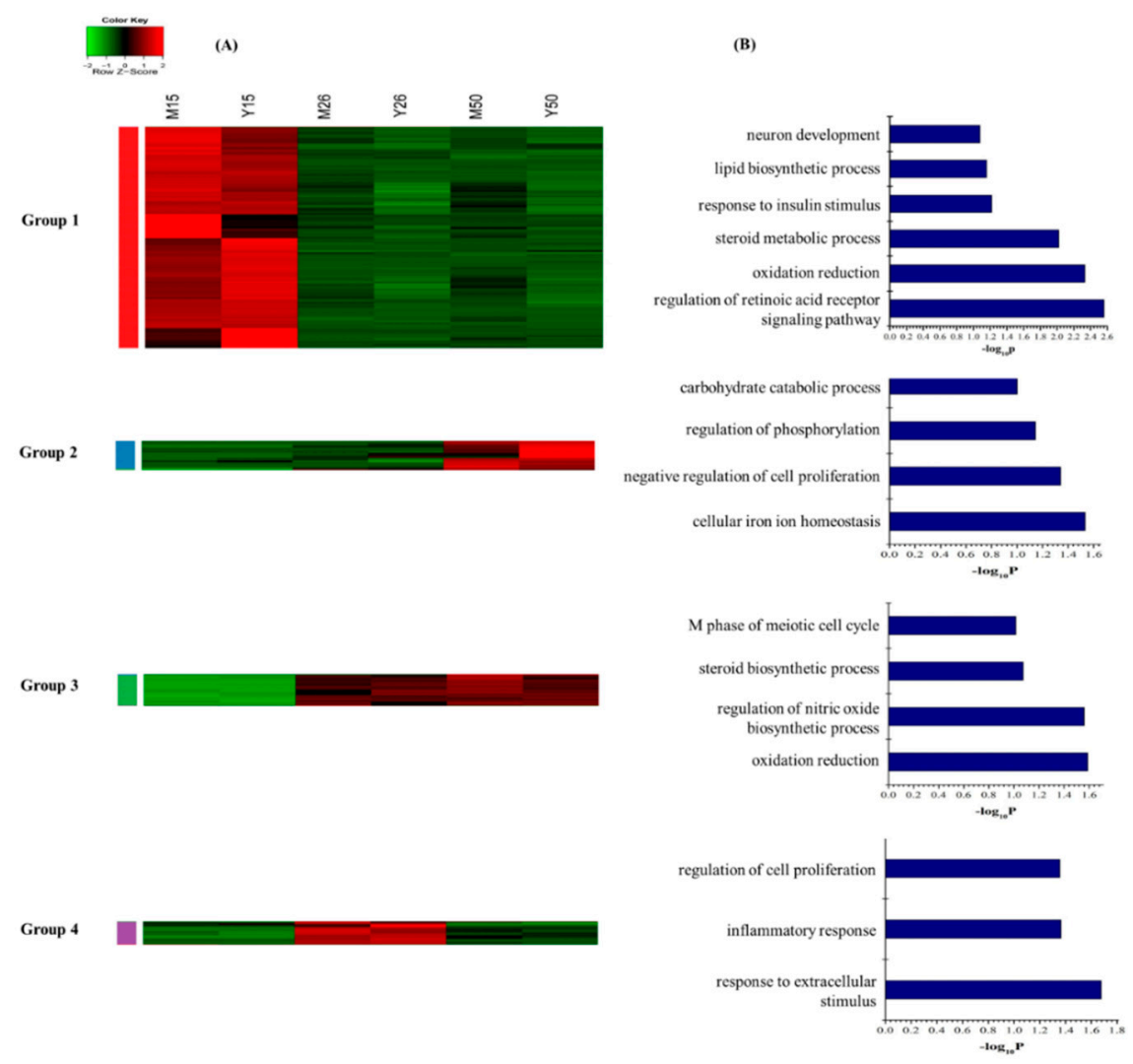

Figure 2. Dynamic progression of the common DEGs in the Meishan and Yorkshire endometrium. (A) Unsupervised hierarchical clustering of the 228 annotated common DEGs in the Meishan and Yorkshire endometrium. The common DEGs were clustered into four groups. Red region, genes up-regulated in the endometrium; green region, genes down-regulated in the endometrium. M15, Meishan pigs on day 15 of gestation; M26, Meishan pigs on day 26 of gestation; M50, Meishan pigs on day 50 of gestation; Y15, Yorkshire pigs on day 15 of gestation; Y26, Yorkshire pigs on day 26 of gestation; Y50, Yorkshire pigs on day 50 of gestation; (B) Functional categories distribution of the common DEGs in the Meishan and Yorkshire endometrium. 
The KEGG pathways with the most representations for the common DEGs were retinol metabolism (genes in group 1), p53 signaling pathway (genes in group 2), androgen and estrogen metabolism (genes in group 3), or steroid hormone biosynthesis (genes in group 4), respectively (Table S7). To further discuss whether these DEGs in corresponding pathway can be mapped on the Cytoscape network, we analyzed the DEGs extracted in each pathway, respectively. A total of 63 out of the 228 annotated common DEGs were mapped on the Cytoscape network in regulation of the retinoic acid receptor signaling pathway (CYP26A1, TRIM16), the integrin-mediated signaling pathway (BCAR1, CEACAM1, EMP2, PXN, CTGF), the sterol biosynthetic process (CH25H, FDFT1, FDPS) and the negative regulation of epithelial cell migration (THBS1, PFN2), respectively (Figure 3).

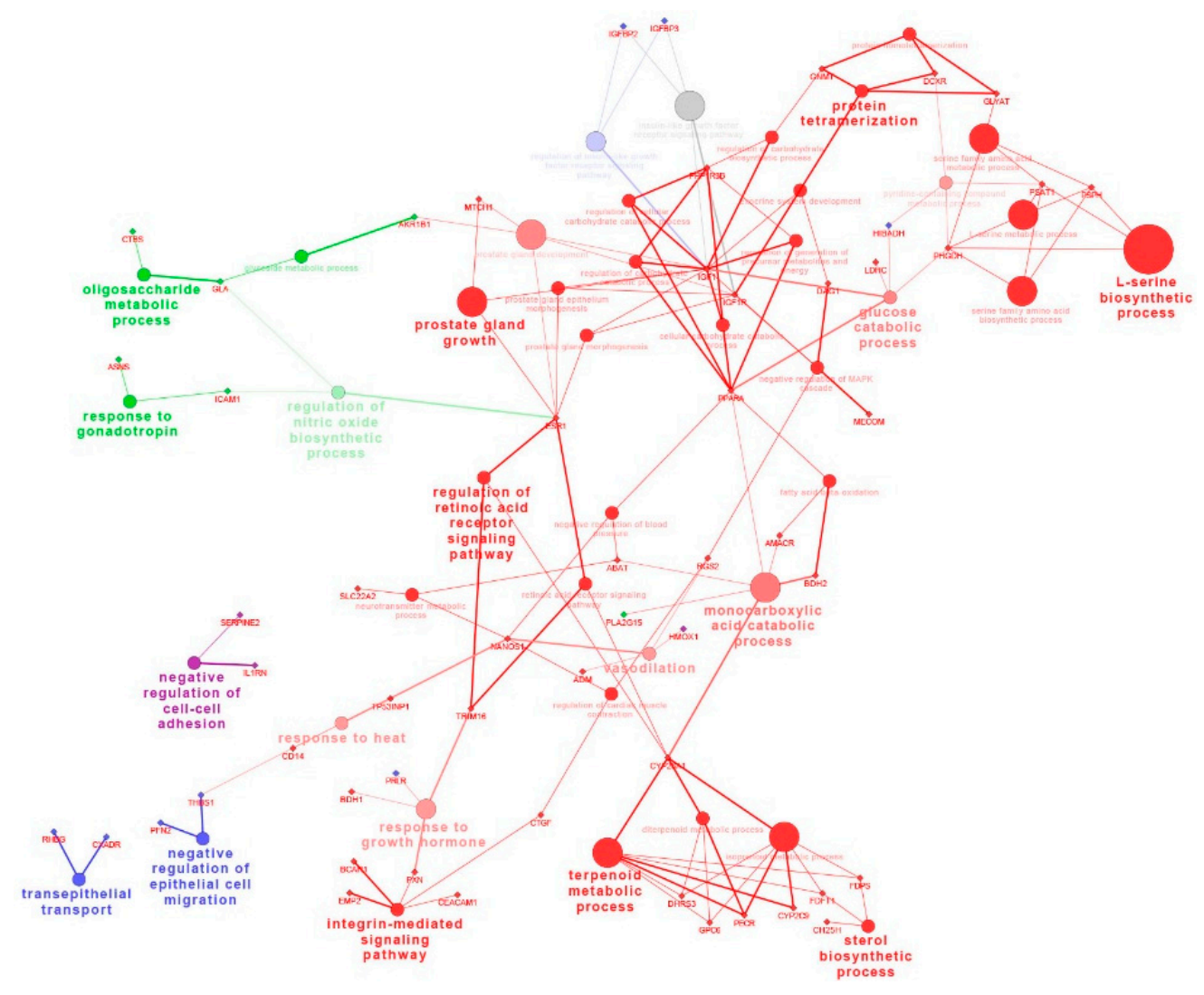

Figure 3. The network of the common DEGs in Meishan and Yorkshire endometrium on gestational day 15, 26 and 50.The genes are represented as diamond. Biological processes are represented as circles. The lines represent the potential connections between the different genes belonging to the different biological process. Red nodes represent genes that have intensive expression on gestational day 15 . Blue nodes represent genes represent genes that have intensive expression on gestational day 50. Green nodes represent genes that have intensive expression on gestational day 26 and 50. Purple nodes represent genes represent genes that have intensive expression on gestational day 26.

\subsection{Pregnancy Causes Distinct Transcriptome Changes between Early and Mid-Gestation in Meishan Endometrium}

Hierarchical clustering based on the 73 unique DEGs in Meishan endometrium displayed four distinct expression patterns (Figure 4A). The group 1 includes the genes with the expression levels increased 
from GD15 to GD26 and decreased at GD50 ( $n=31$ genes). The group 2 includes the genes with the expression levels increased from GD15 to GD26 and then remained constant ( $n=19$ genes). The group 3 consists of the genes with the expression levels decreasing from GD15 to GD26 and then remaining constant ( $n=18$ genes). The expression pattern of the fourth group was that the gene expression levels only increased from GD26 to GD50 ( $n=5$ genes). The enriched GO terms related to the cell cycle were specifically overrepresented for the genes in the group 1. Furthermore, GO terms related to the response to organic substance were present in the group 3 (Figure 4B, Table S8). No significant annotation terms were identified for DEGs in groups 2 and 4. According to the KEGG database, the most significantly enriched pathways were the cell cycle and adherens junction (Table S9). The network analysis showed that PCNA (proliferating cell nuclear antigen), BIRC5 (baculoviral IAP repeat containing 5), MCM4 (minichromosome maintenance complex component) as the candidate genes might play important roles in regulation of Meishan uterine function during the early and mid-gestation (Figure 5).

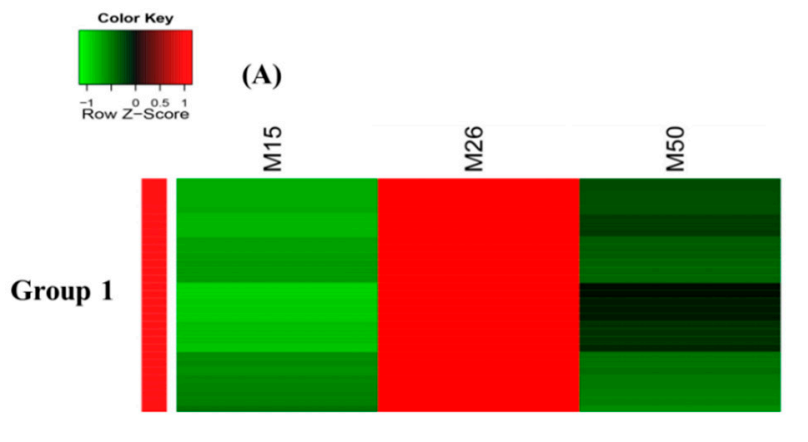

(B)
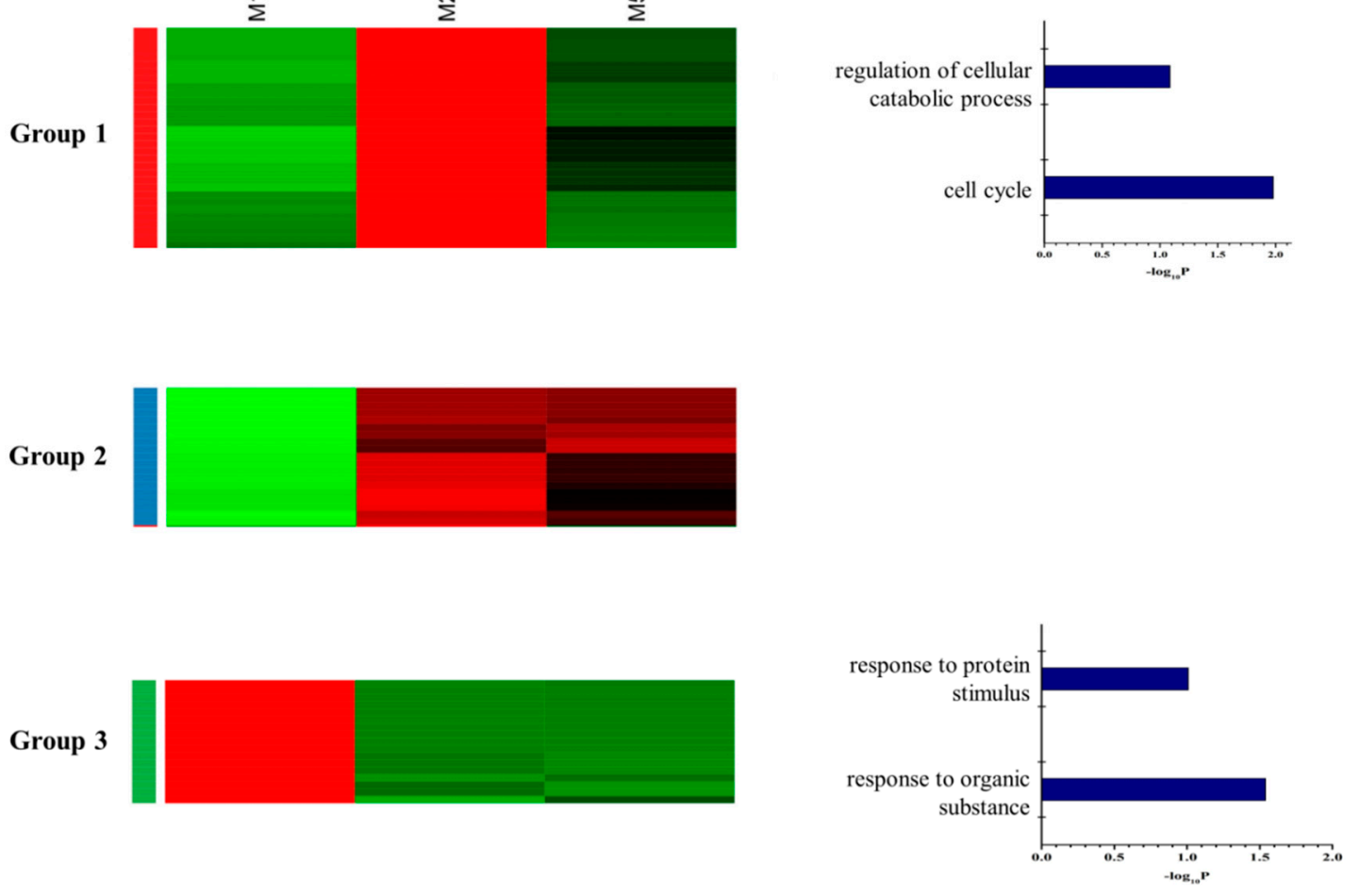

Group 4

Figure 4. Dynamic of unique DEGs in Meishan endometriums. (A) Unsupervised hierarchical clustering of the 73 DEGs unique to Meishan endometrium. DEGs were clustered into four groups. Red region, genes up-regulated in the endometrium, green region, genes down-regulated in the endometrium. M15, Meishan pigs on day 15 of gestation; M26, Meishan pigs on day 26 of gestation; M50, Meishan pigs on day 50 of gestation; (B) Functional categories distribution of the unique DEGs in the Meishan endometrium. 


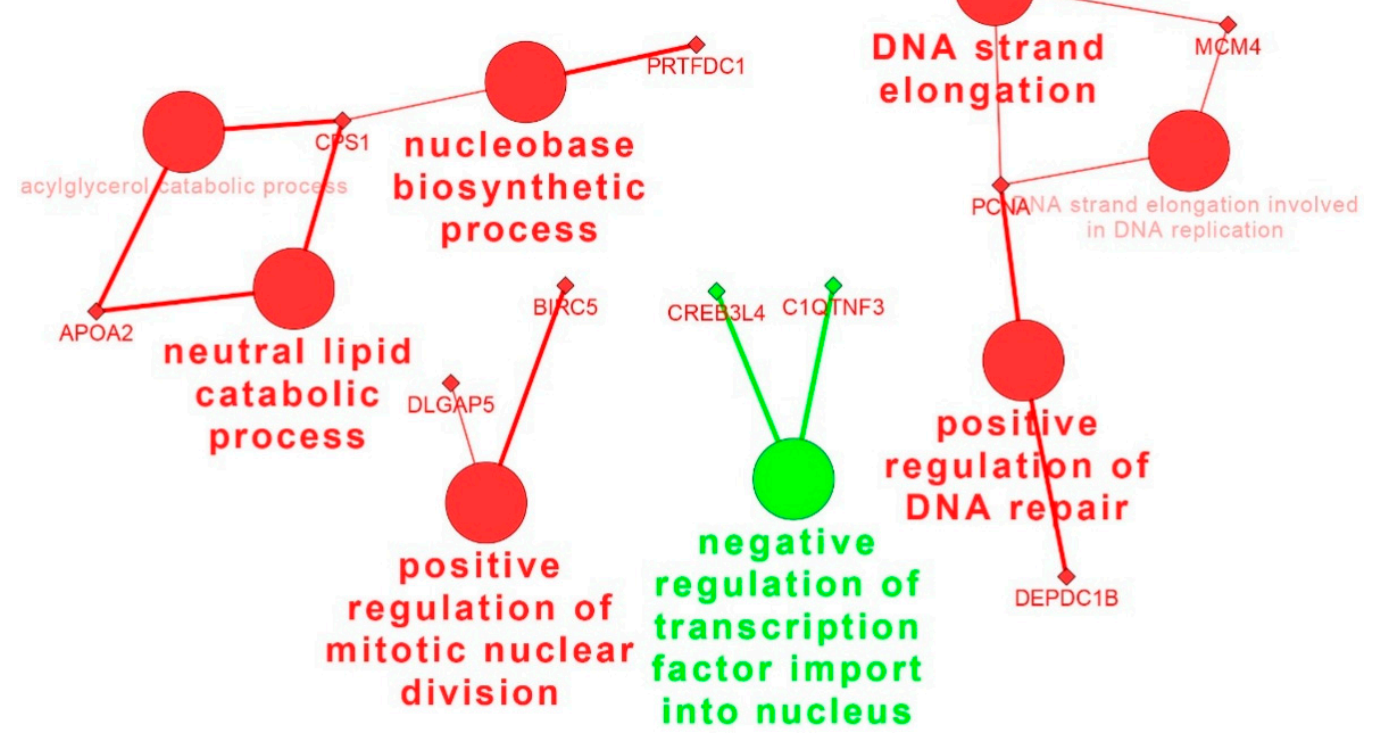

Figure 5. The network of the DEGs unique to Meishan endometrium on gestational days 15 , 26 and 50. The genes are represented as diamond. Biological processes are represented as circles. The lines represent the potential connections between different genes belonging to different biological process. Red nodes represent genes up-regulated on gestational day 26 . Green nodes represent genes highly expressed on gestational day 15.

\subsection{Pregnancy Causes Unique Alteration in the Transcriptome of Yorkshire Endometrium}

Consequently, the 536 DEGs, which were unique in Yorkshire gilts, were classified into four groups by hierarchical clustering analysis (Figure 6A). The first group showed an expression level increased from Day 15 to 26 of gestation then remained unchanged at GD50 ( $n=109$ genes). The second group showed an expression level increased from Day 15 to 26 of gestation and then decreased at GD50 ( $n=56$ genes). The third group showed an expression level decreased from Day 15 to 26 of gestation and thereafter remained constant ( $n=349$ genes). The fourth group showed an expression level increased only from Day 26 to 50 of gestation ( $n=22$ genes). GO terms enriched by the DEGs in the group 1 were associated with regulation of Rho protein signal transduction, maternal placenta development and chordate embryonic development. The genes in the group 2 were related to vasculature development, blastocyst formation and regulation of cell adhesion. The genes in the group 3 were involved in mesodermal cell differentiation, cell proliferation and cell cycle. The genes in the group 4 were involved in chordate embryonic development (Figure 6B, Table S10). As shown in Table S11, the significant signaling pathways include tight junction, regulation of actin cytoskeleton, focal adhesion, MAPK signaling pathway and endocytosis (Table S11). Across the Cytoscape network analysis, the genes in the group 1 were enriched in pathways such as salivary gland morphogenesis and apical junction assembly. The genes in the group 3 were enriched in pathways such as the intracellular estrogen receptor signaling pathway, gland morphogenesis and regulation of Ras GTPase activity (Figure 7). 


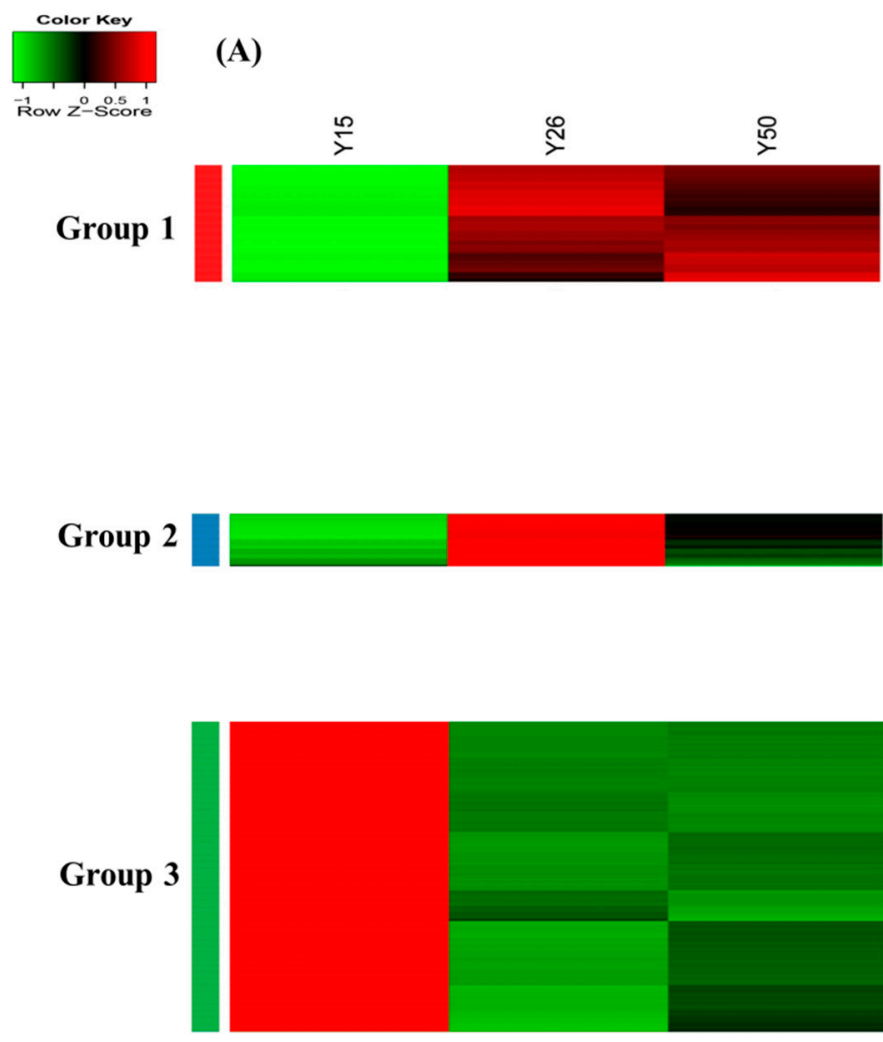

(B)

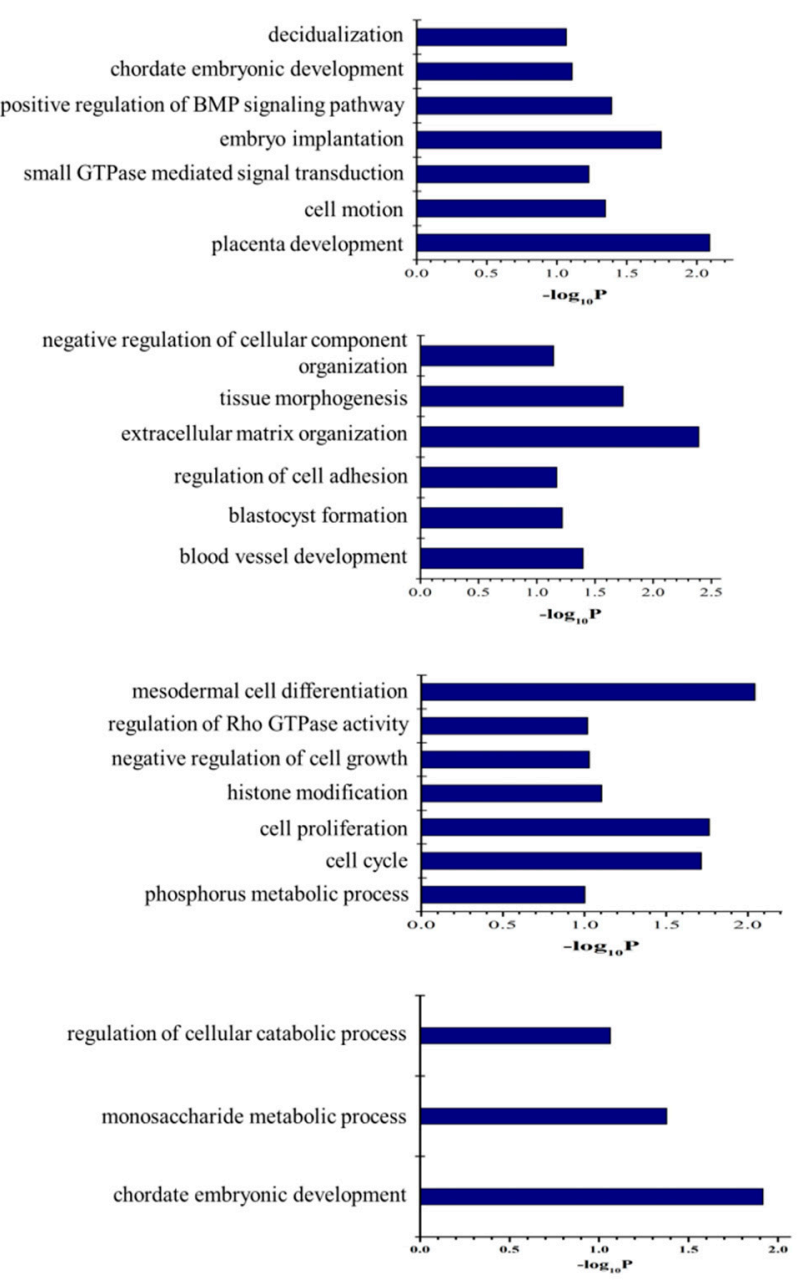

Group 4

Unsupervised hierarchical clustering of 536 differentially expressed genes unique to Yorkshire endometrium. DEGs were clustered into four groups. Red region, genes up-regulated in the endometrium; green region, genes down-regulated in the endometrium. Y15, Yorkshire pigs on day 15 of gestation; Y26, Yorkshire pigs on day 26 of gestation; Y50, Yorkshire pigs on day 50 of gestation; (B) Functional categories distribution of unique DEGs in the Yorkshire endometrium. 


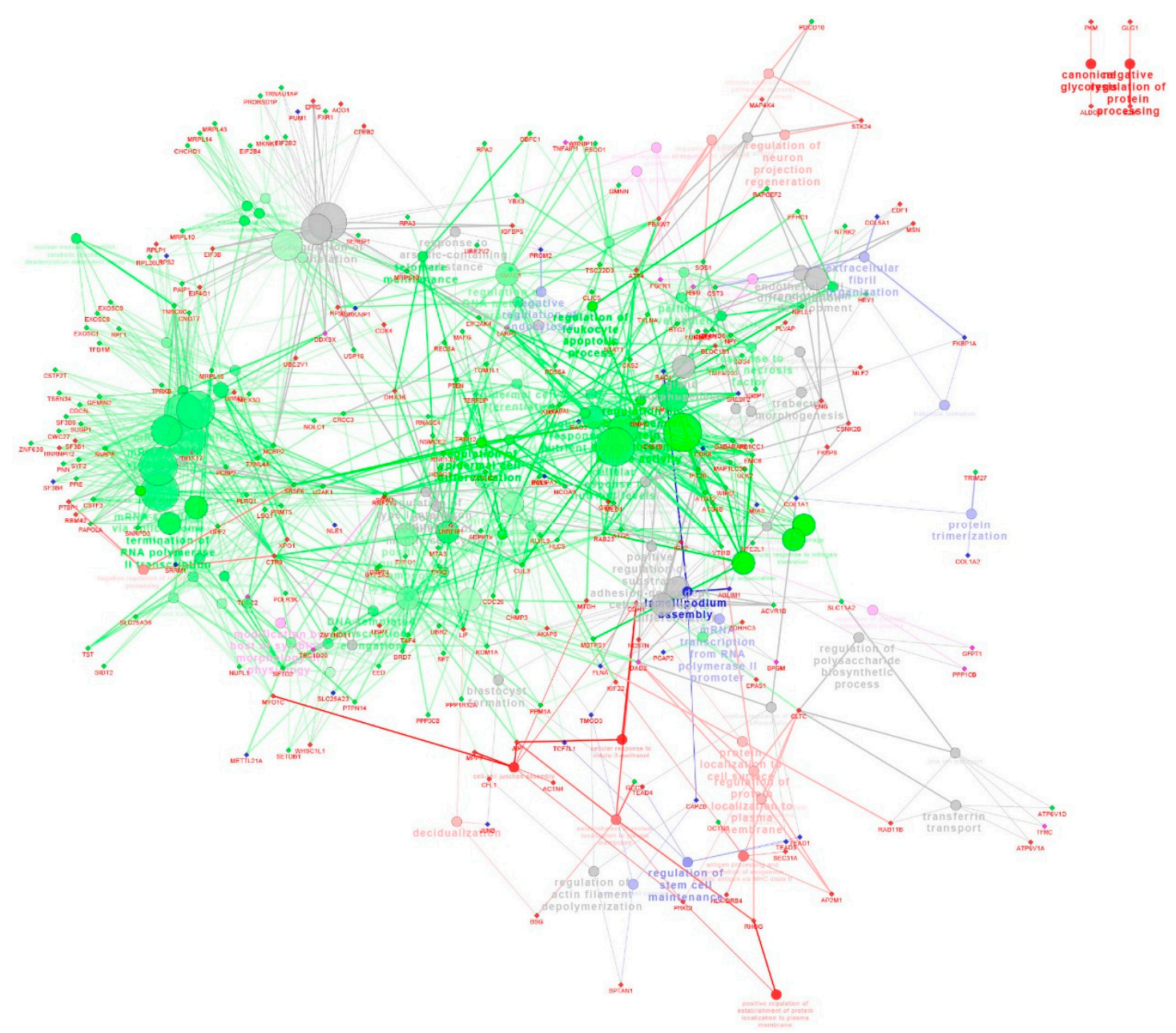

Figure 7. The network of the DEGs unique to Yorkshire endometrium on gestational days 15, 26 and 50. The genes are represented as circles. Biological processes are represented as diamonds. The lines represent the potential regulation relationships between genes or connections between different genes belonging to different biological process. Red nodes represent genes that have intensive expression on gestational day 26 and 50. Blue nodes represent genes that have intensive expression on gestational day 26. Green nodes represent genes that have intensive expression on gestational day 15. Purple nodes represent genes that have intensive expression on gestational day 50 .

\subsection{Verification of the Gene Expression Patterns by Quantitative RT-PCR}

Ten DEGs were selected to validate the results of the microarray analysis by quantitative RT-PCR. Of the selected DEGs, four DEGs were identified as common in Meishan and Yorkshire endometrium (THBS1, HMOX1, PRLR and HSD17B2), one DEG was unique to Meishan endometrium (MSX1), three DEGs were identified as unique to Yorkshire endometrium (STAT1, LIF and BMP4) and two DEGs (MMP7 and S100A9) were expressed differentially in at least one comparison in both Meishan and Yorkshire endometriums. The expression patterns of all the 10 selected DEGs obtained by quantitative RT-PCR were consistent with the results from the microarray analysis (Figure 8). In addition, three DEGs (STC1, ITGB3 and IGFBP3) that showed common expression patterns in Meishan and Yorkshire endometriums but did not reach the statistically significant level in the microarray analysis were also 
selected for validation due to their roles in remodeling of endometrium. The results confirmed the common expression patterns of the three DEGs in Meishan and Yorkshire endometrium (Figure 8).
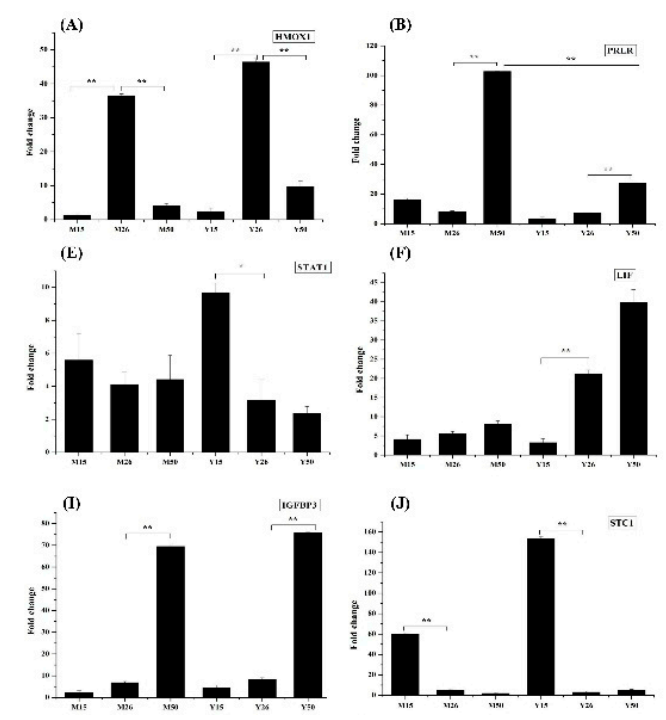
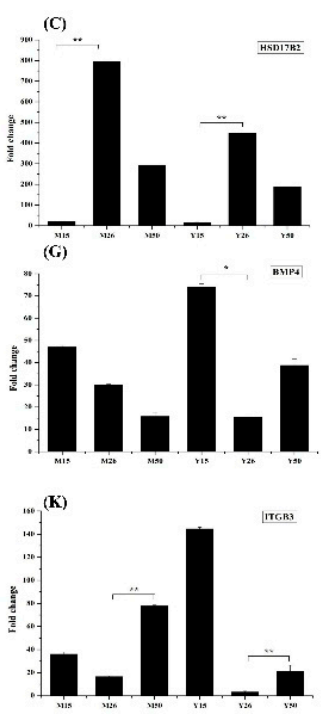
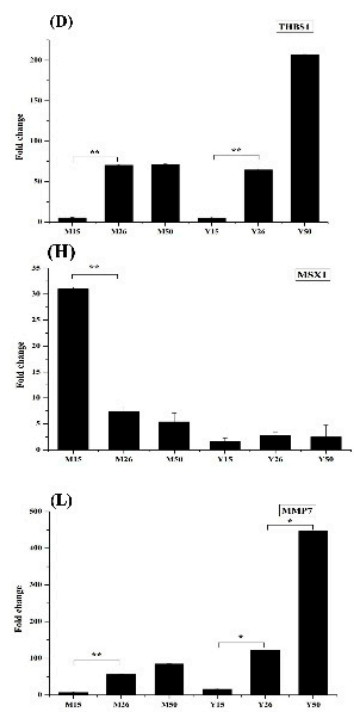

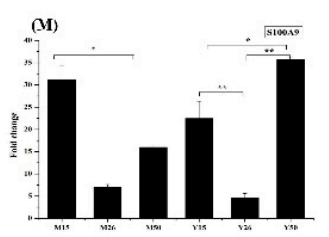

Figure 8. Validation of the expression levels of 13 representative genes by quantitative RT-PCR. The $x$-axis represents the different stages and breeds and the $y$-axis shows the fold changes in expression (** $p<0.01, * p<0.05$ ). (A) HMOXI gene; (B) PRLR gene;

(C) HSD17B2 gene; (D) THBS1 gene; (E) STAT1 gene; (F) LIF gene; (G) BMP4 gene;

(H) MSX1 gene; (I) IGFBP3 gene; (J) STC1 gene; (K) ITGB3 gene; (L) MMP7 gene; (M) $S 100 A 9$ gene.

\section{Discussion}

In this study, the large numbers of DEGs identified in both Meishan and Yorkshire endometriums between GD15 and GD26 (Tables S1 and S2) revealed that major temporal changes occur in the endometrium transcriptome between the conceptus attachment stage (GD15) and the post-implantation stage (GD26). Additionally, we found that the number of DEGs identified as unique to Yorkshire endometrium is much greater compared to the number of DEGs unique to Meishan endometrium, which could suggest that the uterine environment in Meishan breed may be relatively stable.

The endometrium is a complex steroid responsive tissue. The progesterone and estrogen may constitute a "servomechanism" that regulates the endometrial remodeling (remodeling of the endometrial epithelium and stromal cell) and the uterine receptivity during the early pregnancy in pigs [37,38]. In this study, we identified the genes involved in regulation of retinoic acid receptor signaling pathway (ESR1, CYP26A1, TRIM16) and retinol metabolism-related enzyme genes (DHRS3, CYP2C9, CYP26A1) that were highly expressed in both Meishan and Yorkshire endometriums at GD15. It was found that CYP26A1 might block the adverse effect of the retinoic acid in order to promote the successful embryo implantation [39]. TRIM16, also named estrogen-responsive B box protein and acts as a positive regulator of the CYP26A1 expression, showed a similar expression pattern with CYP26A1 gene in both Meishan and Yorkshire endometriums. The expression of ESRI in the porcine endometrium may indicate direct action of estrogen on proliferation and differentiation of endometrial cells [40]. The estrogen and retinoic acid signaling pathway provides regulation of "Yin and Yang" in cell proliferation and survival [38,41]. 
These results reminded us that except the progesterone and estrogen, the interaction between the retinoic acid and estrogen signaling may be also important for the embryo-maternal communications and endometrium remodeling during the early pregnancy.

Interestingly, we found increased expression of genes in steroid hormone biosynthesis pathway (CYP3A7, HSD17B2 and SULT1E1) in GD26 endometrium (Figure 2A, Group 4) and genes encoding androgen and estrogen metabolic enzymes (HSD17B12 and HSD11B1) in both GD26 and GD50 endometrium (Figure 2A, Group 3). These genes play important roles in synthesis and metabolism of progesterone and estrogens. The imbalance between the actions of estrogen and progesterone are associated with abnormal uterine bleeding and proliferative disorders [42]. Sulfotransferases could convert estrogens into non-active estrogen sulphates [33] and the expression of P450 aromatase in the pregnant mouse uterus plays an essential role in converting testosterone into $E_{2}$ [43].Thus, the increased expression of SULTIE1 and CYP3A7 genes in the pregnant endometrium suggested that the regulator to balance the ratio of progesterone to estrogen might be the fine tuning estrogen levels in the uterus. Above all, these results indicated that during the early and mid-gestation, the gene involved in adjusting uterine steroid and retinol concentration may be essential for embryo implantation and growth.

Previous evidence suggests that the endometrial morphology and uterine function differs during the gestational stages in pigs [35]. It has been proposed that the acquisition of the uterine receptivity results from the loss of epithelial cell polarity $[44,45]$. The factors that mainly attribute to the uterine epithelial cell function appear to play a central role in mediating endometrial receptivity. The transcript level of the Msh homeobox 1 gene, Msx 1, was decreased uniquely in Meishan endometrium from GD15 to GD26 and then remained at a low expression level in GD50. As the negative regulator of the cell differentiation, Msxl was expressed in mouse uterine luminal epithelial cell at the time of the pre-implantation phase and declined at time of implantation and post-implantation phases [46-48]. Further investigation indicated that Msxl gene functions in regulation of the transition in uterine luminal epithelial cells from a high to a less polar state and thus leading to a transition of uterus from the pre-receptive to the receptive phase [49]. Thus, compared to Yorkshire pigs, the higher expression of Msxl gene at implantation stage in Meishan endometrium implied a reduced uterine epithelial polarity to make the uterus conducive to blastocyst attachment to the luminal epithelium (LE) at the onset of implantation.

The genes involved in cell cycle, such as CCNB2, NCAPG, DLGAP5, BIRC5, CDC20, TACC1 and $P C N A$, were up-regulated in the post-implantation stage (GD26) and then decreased at GD50 in Meishan endometrium. The cell cycle regulators are critical for controlling the stromal cell proliferation and differentiation during the implantation stage [50].PCNA (proliferating cell nuclear antigen) is a cell cycle promoting gene which was observed primarily in uterine luminal epithelium and glandular epithelium at pre-implantation stage and its expression level was increased gradually in stromal cells and myometrium with progressing gestation [51-53]. This increased expression of cell cycle-related genes at the time of Meishan embryo implantation suggested that the cell proliferation and differentiation in Meishan endometrium were associated with the embryo attachment. Interestingly, gene ontology analysis using group 3 DEGs in Yorkshire endometrium (genes that have intensive expression at GD15) revealed that cell proliferation and cell cycle were among the most significantly enriched biological processes (Figure 6B, Table S10). Compared to group 3 in Yorkshire endometrium (Figure 6) with the group 1 in Meishan endometrium (Figure 4), we found that the cell cycle and cell proliferation regulators showed the opposite expression patterns between Meishan and Yorkshire endometrium during the embryo 
implantation stage (GD15). Taking into account the above, the genes that showed higher expression levels in endomtrium of Meishan pigs on GD15 and GD26 may influence the uterine receptivity by regulating the uterine cell phenotype and contribute to promoting the endometrium remodeling in Meishan pigs during the early pregnancy.

Compared to the Meishan breed, the decreased embryo survival in Yorkshire breed was suggested to be due to a promotive effect of the uterus on embryonic development and growth $[6,54,55]$. We found the genes that were associated with regulation of the maternal placenta development $(L I F, B S G)$ and the chordate embryonic development (FKBP8, EPAS1, TEAD4, CFL1, SPINT1, CDH1) have intensive expression in Yorkshire endometrium at GD26 and GD50.The epithelial marker E-cadherin was found to be localized in luminal and glandular epithelial cells during the peri-implantation period of pregnancy [56]. Its encoding gene, $C D H 1$, was up-regulated from GD15 to GD26 and then remained constant at GD50 (Figure 6). At the same time, we also detected the down-regulated expression of some genes which are involved in suppressing the expression of $C D H 1$ gene, such as the transcriptional repressor of E-cadherin gene (SIP1, also known as ZEB2), and PTEN and GSK3B genes [57,58]. A previous report found that the down-regulation of SIP1 resulted in reduced invasion and migration of cells, along with the up-regulation of $\mathrm{CDH1}$ [59]. The up-regulation of $\mathrm{CDH1}$ also resulted in the increasing of the epithelial cell polarity and glandular formation by inducing mesenchymal-to-epithelial transition (MET) [60-62]. It has been indicated that the polarized uterine epithelial cells could secrete a large number of cytokines and growth factors to promote conceptus elongation and development $[63,64]$. These unique DEGs to Yorkshire endometrium may dynamically regulate uterine epithelial cell polarity, promote secretion of the endometrial gland, thereby stimulating the blastocyst growth and development.

\section{Experimental Section}

\subsection{Animals and Tissue Collection}

All the experimental and surgical procedures complied with the Guide for Care and Use of Laboratory Animals and were approved by the Biological Studies Animal Care and Use Committee of Hubei Province, China. The Meishan and Yorkshire gilts of similar age and weight were observed twice a day for estrous behavior using intact boars. On the day of first standing oestrus (Day 0) and $24 \mathrm{~h}$ later, Meishan and Yorkshire gilts from Jingpin farm of Huazhong Agricultural University were mated to boars of their respective breed. The first day of mating was considered to be Day 0 of gestation. Pregnancy was confirmed by the presence of normal conceptus in the uterine flushing (Day 15) or at hysterectomy (Days 26 and 50). Uteri were obtained from animals slaughtered on Days 15, 26 and 50 of gestation ( $n=2$ gilts//breed/gestational day). Tissue collection were according to the descriptions by $\mathrm{Su}$ et.al (2014) [65]. The endometrial tissues were placed in liquid nitrogen immediately and stored at $-80{ }^{\circ} \mathrm{C}$ until extraction of RNA.

\subsection{Porcine Affymetrix GeneChip Hybridization and Data Analysis}

Total RNA was extracted from the frozen tissues using Trizol reagent (Invitrogen, Carlsbad, CA, USA) according to the manufacturer's instructions. In order to prevent contamination of genomic DNA, RNase-free DNase kit (Qiagen, Valencia, CA, USA) was used according to the manufacturer's manual. 
Total RNA concentrations and quality were determined by using a NanoDrop ND-2000 Spectrophotometer (Thermo Scientific, Wilmington, DE, USA). Then, the amount of each RNA sample used for subsequent microarray analysis was made to be equal in a unit volume. As described by Su et al. (2014) [65], for each breed, RNA sample from one endometrial site of one gilt was pooled in equal volume with that from the corresponding site of the other gilt on each gestational day. Thus, three pools of RNA samples from three different conceptus-attachment sites of two gilts on each gestational day for each breed were used for the microarray hybridization. The RNAs were sent to a commercial service for hybridization to the Porcine AffymetrixGenechip ${ }^{\circledR}$ (Affymetrix, Santa Clara, CA, USA).

The raw data intensity files were read into $\mathrm{R}[66]$ and preprocessed using functions of the Affy and GCRMA packages of the BioConductor project [67]. Probe sets whose intensity was above 100 in at least six arrays were retained and then subjected to identification of the significantly differentially expressed genes by the linear model and the empirical Bayes methods (Limma R package). The genes expressed by the endometrium with significant changes at the three different gestational stages were identified in each breed and the comparisons of GD26 vs. GD15 and GD50 vs. GD26 were made within each breed. The raw $p$-values were adjusted for multiple testing using the Bonferroni and Hochberg false discovery rate methods. Lists of DEGs were selected on the basis of an adjusted $p$-value of $\leq 0.05$ and fold change (FC) $\geq 2$. DEGs were considered to be unique to Meishan (Yorkshire) pigs if they obtained an adjusted $p$-value $<0.05$ and $\mathrm{FC} \geq 2$ in Meishan (Yorkshire) pigs dataset but $p$-value $>0.05$ and $\mathrm{FC}<1.5$ in Yorkshire (Meishan) pigs dataset. Probe annotation was firstly done using the file supplied by Tsai et al. [68]. The target sequences of the probe sets were confirmed by BLAST in porcine genome as well as other genomes including human, mouse and bovine. In total, 23,985 (99\% of all) probe sets were assigned RefSeq annotation. All microarray data have been deposited into the NCBI Gene Expression Omnibus and are accessible through GEO Series accession number GSE51787 [69].

\subsection{Clustering Analysis}

The expression values for the differentially expressed genes obtained by averaging the intensity values of analogous probe sets on the microarray were subjected to unsupervised hierarchical clustering analysis by correlation similarity matrix and complete linkage algorithms using $\mathrm{R}$ language.

\subsection{Gene Ontology (GO) and KEGG Pathway Analysis}

Gene Ontology (GO) enrichment analysis was performed by tools available at DAVID Bioinformatics Resources to detect the overrepresented functional gene categories [70,71]. The list of differentially expressed genes was uploaded into KEGG (Kyoto Encyclopedia of Genes and Genomes) [72] to identify the statistically significantly enriched biological pathways in which the differentially expressed genes participated. The network was built by Cytoscape [73].

\subsection{Quantitative RT-PCR for DEGs Validation}

Quantitative RT-PCR was used to verify the differential expression of 13 genes that were detected by the Affymetrix GeneChip. Total RNA from three endometrial samples from each gilts $(n=4$ gilts/gestational day) was reversely transcribed using SuperScript II Reverse Transcriptase and Oligo(dT) 
(Invitrogen, Carlsbad, CA, USA) according to the manufacturer's instructions. The quantitative RT-PCR was performed on 36 endometrial samples in total (attachment sites/gilts/breeds/gestational days) for each gene to be validated. The primers were listed in Table S12. Each real-time RT-PCR reaction (in $25 \mu \mathrm{L}$ ) contained $2 \times$ SYBR Green Real-time PCR Master Mix (Toyobo, Japan), $0.4 \mu \mathrm{M}$ primers, and $0.5 \mu \mathrm{L}$ of template cDNA. The cycling conditions consisted of an initial, single cycle of 5 min at $95^{\circ} \mathrm{C}$, followed by 40 cycles of $30 \mathrm{~s}$ at $95^{\circ} \mathrm{C}, 30 \mathrm{~s}$ at $60^{\circ} \mathrm{C}, 15 \mathrm{~s}$ at $72^{\circ} \mathrm{C}$, and melting curves were obtained by increasing the temperature from 58 to $95{ }^{\circ} \mathrm{C}$ at $0.5{ }^{\circ} \mathrm{C} / \mathrm{s}$ for $10 \mathrm{~s}$. The PCR amplifications were performed in triplicate for each sample. The LightCycler 480 Software 1.5 (Roche, Risch, canton of Zug, Switzerland) was used to obtain $C_{\mathrm{t}}$ values. Relative quantification analyses were done in EXCEL using the comparative $C_{\mathrm{t}}$ method. The gene expression levels were quantified relative to the expression of $A C T B$ by employing an optimized comparative $C_{\mathrm{t}}\left(\Delta \Delta C_{\mathrm{t}}\right)$ value method. The differences in gene expression levels between groups were compared using the Student's $t$-test. A $p$-value $\leq 0.05$ was considered significant.

\section{Conclusions}

Collectively, the study revealed the common differentially expressed genes in endometrium on days 15, 26 and 50 of gestation in Meishan and Yorkshire pigs which were likely essential for the embryo implantation and placentation in pigs. Findings regarding the genes which were unique to either Meishan or Yorkshire pigs could suggest that different genes or pathways might be involved in endometrial remodeling in two pig breeds during early and mid-gestation.

\section{Supplementary Materials}

Supplementary materials can be found at http://www.mdpi.com/1422-0067/16/09/22692/s1.

\section{Acknowledgments}

This work was supported by the National Basic Research Program of China (2014CB138500), National Natural Science Foundation of China (31272426), National Natural Science Foundation of China (31201785).

\section{Author Contributions}

Jiangnan Huang performed most of the experiments and drafted the paper. Ruize Liu and Lijie Su analyzed the data. Qian Xiao contributed to the collection of samples and performed part of the experiments. Mei Yu designed the experiments and revised the paper.

\section{Conflicts of Interest}

The authors declare no conflict of interest. 


\section{References}

1. Edwards, A.K.; Wessels, J.M.; Kerr, A.; Tayade, C. An overview of molecular and cellular mechanisms associated with porcine pregnancy success or failure. Reprod. Domest. Anim. 2012, 47 (Suppl. 4), 394-401.

2. Kerr, A.; Kridli, R.T.; Khalaj, K.; Wessels, J.M.; Hahnel, A.; Tayade, C. Expression of leptin and its long form receptor at the porcine maternal-fetal interface: Contrasting healthy and arresting conceptus attachment sites during early and mid-pregnancy. Reprod. Biol. Endocrinol. 2014, 12, doi:10.1186/1477-7827-12-91.

3. Galvin, J.M.; Wilmut, I.; Day, B.N.; Ritchie, M.; Thomson, M.; Haley, C.S. Reproductive performance in relation to uterine and embryonic traits during early gestation in Meishan, large white and crossbred sows. J. Reprod. Fertil. 1993, 98, 377-384.

4. Biensen, N.J.; Wilson, M.E.; Ford, S.P. The impacts of uterine environment and fetal genotype on conceptus size and placental vascularity during late gestation in pigs. J. Anim. Sci. 1999, 77, 954-959.

5. Youngs, C.R.; Ford, S.P.; McGinnis, L.K.; Anderson, L.H. Investigations into the control of litter size in swine: I. Comparative studies on in vitro development of Meishan and Yorkshire preimplantation embryos. J. Anim. Sci. 1993, 71, 1561-1565.

6. Youngs, C.R.; Christenson, L.K.; Ford, S.P. Investigations into the control of litter size in swine: III. A reciprocal embryo transfer study of early conceptus development. J. Anim. Sci. 1994, 72, 725-731.

7. Christenson, R.K.; Leymaster, K.A.; Young, L.D. Justification of unilateral hysterectomy-ovariectomy as a model to evaluate uterine capacity in swine. J. Anim. Sci. 1987, 65, 738-744.

8. Bazer, F.W.; Thatcher, W.W.; Matinat-Botte, F.; Terqui, M.; Lacroix, M.C.; Bernard, S.; Revault, M.; Dubois, D.H. Composition of uterine flushings from Large White and prolific Chinese Meishan gilts. Reprod. Fertil. Dev. 1991, 3, 51-60.

9. Anderson, L.H.; Christenson, L.K.; Christenson, R.K.; Ford, S.P. Investigations into the control of litter size in swine: II. Comparisons of morphological and functional embryonic diversity between Chinese and American breeds. J. Anim. Sci. 1993, 71, 1566-1571.

10. Spencer, T.E.; Bazer, F.W. Uterine and placental factors regulating conceptus growth in domestic animals. J. Anim. Sci. 2004, 82 (E-Suppl.), E4-E13.

11. Vonnahme, K.A.; Ford, S.P. Differential expression of the vascular endothelial growth factor-receptor system in the gravid uterus of yorkshire and Meishan pigs. Biol. Reprod. 2004, 71, 163-169.

12. Ostrup, E.; Bauersachs, S.; Blum, H.; Wolf, E.; Hyttel, P. Differential endometrial gene expression in pregnant and nonpregnant sows. Biol. Reprod. 2010, 83, 277-285.

13. Stroband, H.W.; van der Lende, T. Embryonic and uterine development during early pregnancy in pigs. J. Reprod. Fertil. Suppl. 1990, 40, 261-277.

14. Thie, M.; Denker, H.W. In vitro studies on endometrial adhesiveness for trophoblast: Cellular dynamics in uterine epithelial cells. Cells Tissues Organs 2002, 172, 237-252.

15. Burghardt, R.C.; Bowen, J.A.; Newton, G.R.; Bazer, F.W. Extracellular matrix and the implantation cascade in pigs. J. Reprod. Fertil. Suppl. 1997, 52, 151-164.

16. Igwebuike, U.M. A review of uterine structural modifications that influence conceptus implantation and development in sheep and goats. Anim. Reprod. Sci. 2009, 112, 1-7. 
17. Bazer, F.W.; Vallet, J.L.; Roberts, R.M.; Sharp, D.C.; Thatcher, W.W. Role of conceptus secretory products in establishment of pregnancy. J. Reprod. Fertil. 1986, 76, 841-850.

18. Geisert, R.D.; Zavy, M.T.; Moffatt, R.J.; Blair, R.M.; Yellin, T. Embryonic steroids and the establishment of pregnancy in pigs. J. Reprod. Fertil. Suppl. 1990, 40, 293-305.

19. Marcelo, P.; Lefevre, F. Induction of the IFN-gamma gene and protein is linked to the establishment of cell polarity in a porcine epithelial cell line. Exp. Cell Res. 2002, 280, 33-44.

20. Bazer, F.W.; Burghardt, R.C.; Johnson, G.A.; Spencer, T.E.; Wu, G. Interferons and progesterone for establishment and maintenance of pregnancy: Interactions among novel cell signaling pathways. Reprod. Biol. 2008, 8, 179-211.

21. Bazer, F.W.; Wu, G.; Spencer, T.E.; Johnson, G.A.; Burghardt, R.C.; Bayless, K. Novel pathways for implantation and establishment and maintenance of pregnancy in mammals. Mol. Hum. Reprod. 2010, 16, 135-152.

22. Fenton, F.R.; Schwartz, F.L.; Bazer, F.W.; Robison, O.W.; Ulberg, L.C. Stage of gestation when uterine capacity limits embryo survival in gilts. J. Anim. Sci. 1972, 35, 383-388.

23. Zygmunt, M.; Herr, F.; Munstedt, K.; Lang, U.; Liang, O.D. Angiogenesis and vasculogenesis in pregnancy. Eur J. Obstet. Gynecol. Reprod. Biol. 2003, 110 (Suppl. 1), S10-S18.

24. Dantzer, V. Electron microscopy of the initial stages of placentation in the pig. Anat. Embryol. 1985, 172, 281-293.

25. Simmen, R.C.; Simmen, F.A.; Ko, Y.; Bazer, F.W. Differential growth factor content of uterine luminal fluids from large white and prolific Meishan pigs during the estrous cycle and early pregnancy. J. Anim. Sci. 1989, 67, 1538-1545.

26. Simmen, F.A.; Simmen, R.C.; Geisert, R.D.; Martinat-Botte, F.; Bazer, F.W.; Terqui, M. Differential expression, during the estrous cycle and pre- and postimplantation conceptus development, of messenger ribonucleic acids encoding components of the pig uterine insulin-like growth factor system. Endocrinology 1992, 130, 1547-1556.

27. Vallee, M.; Beaudry, D.; Roberge, C.; Matte, J.J.; Blouin, R.; Palin, M.F. Isolation of differentially expressed genes in conceptuses and endometrial tissue of sows in early gestation. Biol. Reprod. 2003, 69, 1697-1706.

28. Zhang, H.; Wang, S.; Liu, M.; Zhang, A.; Wu, Z.; Zhang, Z.; Li, J. Differential gene expression in the endometrium on gestation day 12 provides insight into sow prolificacy. BMC Genom. 2013, 14 , doi:10.1186/1471-2164-14-45.

29. Samborski, A.; Graf, A.; Krebs, S.; Kessler, B.; Reichenbach, M.; Reichenbach, H.D.; Ulbrich, S.E.; Bauersachs, S. Transcriptome changes in the porcine endometrium during the preattachment phase. Biol. Reprod. 2013, 89, doi:10.1095/biolreprod.113.112177.

30. Ka, H.; Seo, H.; Kim, M.; Choi, Y.; Lee, C.K. Identification of differentially expressed genes in the uterine endometrium on day 12 of the estrous cycle and pregnancy in pigs. Mol. Reprod. Dev. 2009, $76,75-84$.

31. Samborski, A.; Graf, A.; Krebs, S.; Kessler, B.; Bauersachs, S. Deep sequencing of the porcine endometrial transcriptome on day 14 of pregnancy. Biol. Reprod. 2013, 88, doi:10.1095/biolreprod.113.107870. 
32. Gu, T.; Zhu, M.J.; Schroyen, M.; Qu, L.; Nettleton, D.; Kuhar, D.; Lunney, J.K.; Ross, J.W.; Zhao, S.H.; Tuggle, C.K. Endometrial gene expression profiling in pregnant Meishan and Yorkshire pigs on day 12 of gestation. BMC Genom. 2014, 15, doi:10.1186/1471-2164-15-156.

33. Franczak, A.; Wojciechowicz, B.; Kotwica, G. Transcriptomic analysis of the porcine endometrium during early pregnancy and the estrous cycle. Reprod. Biol. 2013, 13, 229-237.

34. Kiewisz, J.; Krawczynski, K.; Lisowski, P.; Blitek, A.; Zwierzchowski, L.; Ziecik, A.J.; Kaczmarek, M.M. Global gene expression profiling of porcine endometria on Days 12 and 16 of the estrous cycle and pregnancy. Theriogenology 2014, 82, 897-909.

35. Christenson, R.K.; Vallet, J.L.; Leymaster, K.A.; Young, L.D. Uterine function in Meishan pigs. J. Reprod. Fertil. Suppl. 1993, 48, 279-289.

36. Hong, L.; Hou, C.; Li, X.; Li, C.; Zhao, S.; Yu, M. Expression of heparanase is associated with breed-specific morphological characters of placental folded bilayer between Yorkshire and Meishan pigs. Biol. Reprod. 2014, 90, doi:10.1095/biolreprod.113.114181.

37. Ziecik, A.J.; Waclawik, A.; Kaczmarek, M.M.; Blitek, A.; Jalali, B.M.; Andronowska, A. Mechanisms for the establishment of pregnancy in the pig. Reprod. Domest. Anim. 2011, 46 (Suppl. 3), 31-41.

38. Hua, S.; Kittler, R.; White, K.P. Genomic antagonism between retinoic acid and estrogen signaling in breast cancer. Cell 2009, 137, 1259-1271.

39. Ma, J.J.; Han, B.C.; Yang, Y.; Peng, J.P. Retinoic acid synthesis and metabolism are concurrent in the mouse uterus during peri-implantation. Cell Tissue Res. 2012, 350, 525-537.

40. Knapczyk-Stwora, K.; Durlej, M.; Duda, M.; Czernichowska-Ferreira, K.; Tabecka-Lonczynska, A.; Slomczynska, M. Expression of oestrogen receptor alpha and oestrogen receptor beta in the uterus of the pregnant swine. Reprod. Domest. Anim. 2011, 46, 1-7.

41. Ombra, M.N.; Di Santi, A.; Abbondanza, C.; Migliaccio, A.; Avvedimento, E.V.; Perillo, B. Retinoic acid impairs estrogen signaling in breast cancer cells by interfering with activation of LSD1 via PKA. Biochim. Biophys. Acta 2013, 1829, 480-486.

42. Lessey, B.A. Fine tuning of endometrial function by estrogen and progesterone through microRNAs. Biol. Reprod. 2010, 82, 653-655.

43. Das, A.; Mantena, S.R.; Kannan, A.; Evans, D.B.; Bagchi, M.K.; Bagchi, I.C. De novo synthesis of estrogen in pregnant uterus is critical for stromal decidualization and angiogenesis. Proc. Natl. Acad. Sci. USA 2009, 106, 12542-12547.

44. Thie, M.; Fuchs, P.; Denker, H.W. Epithelial cell polarity and embryo implantation in mammals. Int. J. Dev. Biol. 1996, 40, 389-393.

45. Denker, H.W. Endometrial receptivity: Cell biological aspects of an unusual epithelium. A review. Ann. Anat. Anat. Anz. 1994, 176, 53-60.

46. Mansouri-Attia, N.; Aubert, J.; Reinaud, P.; Giraud-Delville, C.; Taghouti, G.; Galio, L.; Everts, R.E.; Degrelle, S.; Richard, C.; Hue, I.; et.al. Gene expression profiles of bovine caruncular and intercaruncular endometrium at implantation. Physiol. Genom. 2009, 39, 14-27.

47. Pavlova, A.; Boutin, E.; Cunha, G.; Sassoon, D. Msx1 (Hox-7.1) in the adult mouse uterus: Cellular interactions underlying regulation of expression. Development 1994, 120, 335-345.

48. Daikoku, T.; Cha, J.; Sun, X.; Tranguch, S.; Xie, H.; Fujita, T.; Hirota, Y.; Lydon, J.; DeMayo, F.; Maxson, R.; Dey, S.K. Conditional deletion of Msx homeobox genes in the uterus inhibits blastocyst implantation by altering uterine receptivity. Dev. Cell 2011, 21, 1014-1025. 
49. Nallasamy, S.; Li, Q.; Bagchi, M.K.; Bagchi, I.C. Msx homeobox genes critically regulate embryo implantation by controlling paracrine signaling between uterine stroma and epithelium. PLoS Genet. 2012, 8, e1002500.

50. Das, S.K. Cell cycle regulatory control for uterine stromal cell decidualization in implantation. Reproduction 2009, 137, 889-899.

51. Lai, M.D.; Lee, L.R.; Cheng, K.S.; Wing, L.Y. Expression of proliferating cell nuclear antigen in luminal epithelium during the growth and regression of rat uterus. J. Endocrinol. 2000, 166, 87-93.

52. Oner, H.; Oner, J.; Demir, R. Distributions of PCNA and Cas-3 in rat uterus during early pregnancy. Folia. Histochem. Cytobiol. 2010, 48, 71-77.

53. Korgun, E.T.; Celik-Ozenci, C.; Acar, N.; Cayli, S.; Desoye, G.; Demir, R. Location of cell cycle regulators cyclin B1, cyclin A, PCNA, Ki67 and cell cycle inhibitors p21, p27 and p57 in human first trimester placenta and deciduas. Histochem. Cell Biol. 2006, 125, 615-624.

54. Vonnahme, K.A.; Wilson, M.E.; Ford, S.P. Conceptus competition for uterine space: Different strategies exhibited by the Meishan and Yorkshire pig. J. Anim. Sci. 2002, 80, 1311-1316.

55. Wilson, M.E.; Biensen, N.J.; Youngs, C.R.; Ford, S.P. Development of Meishan and Yorkshire littermate conceptuses in either a Meishan or Yorkshire uterine environment to day 90 of gestation and to term. Biol. Reprod. 1998, 58, 905-910.

56. Kiewisz, J.; Kaczmarek, M.M.; Andronowska, A.; Blitek, A.; Ziecik, A.J. Gene expression of WNTs, beta-catenin and E-cadherin during the periimplantation period of pregnancy in pigs-involvement of steroid hormones. Theriogenology 2011, 76, 687-699.

57. Mao, Y.; Xu, J.; Li, Z.; Zhang, N.; Yin, H.; Liu, Z. The Role of Nuclear beta-Catenin Accumulation in the Twist2-Induced Ovarian Cancer EMT. PLoS ONE 2013, 8, e78200.

58. Bae, G.Y.; Choi, S.J.; Lee, J.S.; Jo, J.; Lee, J.; Kim, J.; Cha, H.J. Loss of E-cadherin activates EGFR-MEK/ERK signaling, which promotes invasion via the ZEB1/MMP2 axis in non-small cell lung cancer. Oncotarget 2013, 4, 2512-2522.

59. Nakamura, K.; Hongo, A.; Kodama, J.; Hiramatsu, Y. The role of hepatocyte growth factor activator inhibitor (HAI)-1 and HAI-2 in endometrial cancer. Int. J. Cancer 2011, 128, 2613-2624.

60. Reardon, S.N.; King, M.L.; MacLean, J.A., 2nd; Mann, J.L.; DeMayo, F.J.; Lydon, J.P.; Hayashi, K. CDH1 is essential for endometrial differentiation, gland development, and adult function in the mouse uterus. Biol. Reprod. 2012, 86, 1-10.

61. Auersperg, N.; Pan, J.; Grove, B.D.; Peterson, T.; Fisher, J.; Maines-Bandiera, S.; Somasiri, A.; Roskelley, C.D. E-cadherin induces mesenchymal-to-epithelial transition in human ovarian surface epithelium. Proc. Natl. Acad. Sci. USA 1999, 96, 6249-6254.

62. Bhatt, T.; Rizvi, A.; Batta, S.P.; Kataria, S.; Jamora, C. Signaling and mechanical roles of E-cadherin. Cell Commun. Adhes. 2013, 20, 189-99.

63. Filant, J.; Spencer, T.E. Uterine glands: Biological roles in conceptus implantation, uterine receptivity and decidualization. Int. J. Dev. Biol. 2014, 58, 107-116.

64. Fahey, J.V.; Schaefer, T.M.; Channon, J.Y.; Wira, C.R. Secretion of cytokines and chemokines by polarized human epithelial cells from the female reproductive tract. Hum. Reprod. 2005, 20, 1439-1446.

65. Su, L.; Liu, R.; Cheng, W.; Zhu, M.; Li, X.; Zhao, S.; Yu, M. Expression patterns of microRNAs in porcine endometrium and their potential roles in embryo implantation and placentation. PLoS ONE 2014, 9, e87867. 
66. The R Project for Statistical Computing. Available online: https://www.r-project.org (accessed on 13 April 2015).

67. Bioconductor. Available online: http://www.bioconductor.org (accessed on 13 April 2015).

68. Tsai, S.; Cassady, J.P.; Freking, B.A.; Nonneman, D.J.; Rohrer, G.A.; Piedrahita, J.A. Annotation of the Affymetrix porcine genome microarray. Anim. Genet. 2006, 37, 423-424.

69. GEO Series accession number GSE51787. Available online: http://www.ncbi.nlm.nih.gov/ geo/query/acc.cgi?acc=GSE51787 (accessed on 16 September 2015).

70. Huang da, W.; Sherman, B.T.; Lempicki, R.A. Systematic and integrative analysis of large gene lists using DAVID bioinformatics resources. Nat. Protoc. 2009, 4, 44-57.

71. Zhang, W.; Huang, R.S.; Duan, S.; Dolan, M.E. Gene set enrichment analyses revealed differences in gene expression patterns between males and females. Silico Biol. 2009, 9, 55-63.

72. Kanehisa, M.; Goto, S.; Sato, Y.; Furumichi, M.; Tanabe, M. KEGG for integration and interpretation of large-scale molecular data sets. Nucleic Acids Res. 2012, 40, D109-D114.

73. Cytoscape. Available online: http://www.cytoscape.org (accessed on 25 May 2015).

(C) 2015 by the authors; licensee MDPI, Basel, Switzerland. This article is an open access article distributed under the terms and conditions of the Creative Commons Attribution license (http://creativecommons.org/licenses/by/4.0/). 